\title{
RNA extraction alternative method for SARS-CoV-2 molecular diagnosis
}

\author{
Elisa Bianchini ${ }^{1}{ }^{\#}$, Francesco Rossignolo $^{1}{ }^{\#}$, Melissa Perfranceschi $^{1}$, Chiara Cazzin ${ }^{1}$, Ronaldo Silva ${ }^{2}$, Fabio Formenti ${ }^{2}$ \\ Francesca Perandin ${ }^{2}$, Cristiana Griffante', Rosaria Remelli', Michela Padovani' ${ }^{2}$, Salvatore Scarso ${ }^{2}$, Martina \\ Leonardi ${ }^{2}$ and Chiara Piubelli ${ }^{2, *}$ \\ ${ }^{1}$ Aptuit Srl, an Evotec Company, Verona, Italy \\ 2 Department of Infectious-Tropical Diseases and Microbiology, IRCCS Sacro Cuore Don Calabria Hospital, \\ Negrar, Italy \\ \# Equal contribution \\ * Correspondence: chiara.piubelli@sacrocuore.it (CP)
}

\begin{abstract}
Background: the devastating outbreak of COVID-19 poses serious challenges for the diagnostics laboratories, which are often facing global shortage of reagents and equipment. With the aim of increasing the diagnostic throughput for SARS-CoV-2 molecular test, the purpose of this study was to validate an additional RNA extraction method respect to those already recommended by WHO and the US Centers for Disease Control and Prevention (CDC). Methods: a new protocol for RNA extraction from nasopharyngeal swab was set up, adapting the Qiagen RNeasy 96 plate and validated on a set of 100 clinical samples analyzed in parallel by Roche-Magnapure method (already recommended by CDC guidelines). Results: the internal control and target genes analysis showed a good agreement between the two extraction methods indicating that the two methods can be considered equivalent and that the RNeasy-adapted method can be applied for the SARS-CoV-2 diagnostics. The addition of this new extraction method resulted in a throughput increase for SARSCoV-2 molecular test of about 2000 samples/month during the initial months of the pandemic emergency in which the lack of reagents for the extraction led to an insufficient sample processing throughput of the analysis of the swabs.
\end{abstract}

Keywords: COVID-19, SARS-CoV-2, nasopharyngeal swab, RNA extraction, RT-PCR.

\section{Introduction}

From the beginning of the epidemic in January 2020, the severe acute respiratory syndrome coronavirus 2 (SARS-CoV-2) reached almost every country worldwide, causing to date 159,188,745 global confirmed cases of COVID-19, and 3,308,588 deaths [World Health Organization (WHO) dashboard data, at 10 ${ }^{\mathrm{TH}}$ May 2021, https://covid19.who.int/]. The devastating outbreak tragically overwhelmed health-care systems in most countries, with serious challenges also for the diagnostics laboratories, due to a global shortage of reagents and equipment, which is still a problem as demonstrated in a recent survey conducted by the American Society for Microbiology (https://asm.org/Articles/2020/September/Clinical-Microbiology-Supply-Shortage-Collecti-1). Currently, the most accurate diagnostic test to confirm Coronavirus Disease 2019 (COVID-19) clinical evaluation is realtime reverse transcription polymerase chain reaction (rRT-PCR) test on respiratory specimens (notably nasopharyngeal swab, sputum, broncho alveolar lavage fluid). Both WHO and the US Centers for Disease Control and Prevention (CDC) produced general guidelines with several different recommended protocols for specimens handling, RNA extraction and rRT-PCR for SARS-CoV-2 molecular test [1,2]. Notwithstanding the recent development of extraction-free protocols [3-6], due to the presence of inhibitory agents in the starting material [7], methods with RNA extraction still maintain the highest sensitivity, 
and are the recommended methods for SARS-CoV-2 molecular diagnosis [https://www.cdc.gov/coronavirus/2019-ncov/lab/testing.html]. Currently, the different rRT-PCR available for diagnostic purposes are targeting six genes of SARS-CoV-2 RNA: ORF1a/b, ORF1b-nsp14 (50-UTR), RNA-dependent RNA polymerase (RdRp), spike protein $(S)$, envelope (E), nucleocapsid protein $(\mathrm{N})$ and RNA-dependent RNA polymerase/helicase (RdRp/Hel). Notably, nucleocapsid N2 and E genes were found to be the most sensitive singleplex reactions and no significant change in cycle threshold $(\mathrm{Ct})$ was noted when both assays were combined [8-10]. FDA-approved CDC 2019-Novel Coronavirus (2019-nCoV) rRT-PCR Diagnostic Panel, targets two regions of the N gene (N1 and N2) [https://www.fda.gov/media/134922/download].

Despite the recommendation on a set of validated methods for RNA extraction and rRTPCR, due to the serious emergency conditions and lack of reagents, the Food and Drug Administration (FDA) admitted the use of alternative methods, in addition to the Emergency Use-Authorized (EUA) test, where the modified test was validated using a bridging study to the EUA test.

Italy has been one of the most severely hit countries by the COVID-19 [11]. Since the beginning of the outbreak, the Department of Infectious, Tropical Diseases and Microbiology (DITM) of the IRCCS Sacro Cuore Don Calabria Hospital was involved in a massive diagnostic analysis of respiratory specimens from COVID-19 suspected patients. Despite the medium size of the Hospital, from March 2020 to April 2021, DITM analyzed about 120000 samples, not only from patients coming to the emergency room of our hospital but also from subjects of the local province. As for many other hospitals, our laboratory faced the problem of reagents scarcity, in particular for RNA extraction procedure, which is still a critical issue for the current diagnostic need.

A new protocol for RNA extraction from nasopharyngeal swab was set up, using an alternative method respect to the routinely used method, with the aim of increasing the diagnostic throughput for SARS-CoV-2 molecular test. The new method was not included among the EUA tests recommended by CDC. For this reason, the aim of the described study was to validate the additional extraction method on clinical samples, through a comparison with the routinely used EUA-diagnostic method.

\section{Results and Discussion}

During the pandemic emergency, due to reagent scarcity, our laboratory decided to validate an alternative method for RNA extraction to support SARS-CoV-2 molecular diagnostics. Among the extraction kits recommended by the CDC for viral RNA extraction to diagnose SARS-CoV-2 infection, the Qiagen RNeasy kit was not included. The only study reporting the possible application of this kit for SARS-CoV-2 diagnosis, was a pre-print paper of Bruce and colleagues [4], in which the kit was analytically compared to the QIAamp Viral Mini (Qiagen) kit by a spiking experiment but the paper lacks of a validation on clinical samples.

In the present study, a comparison between the Magnapure LC RNA isolation kit (already included in the CDC panel), used as reference, and the RNeasy $96 \mathrm{kit}$, applied as alternative method, has been performed. The new alternative protocol for RNA extraction was adapted from the RNeasy protocol utilized for cell lines. Differently from the routinely used method, it is based on column absorption instead of magnetic beads separation and the procedure is mainly manual instead of being an automated method. The alternative method, adapted for the nasopharyngeal swab matrix, was validated on a set of 96 clinical samples (18 positive and 78 negative samples to SARS-CoV-2 detection using the routine method). A sample was considered positive when at least one of the two SARS-CoV-2 specific targets (N1 or N2) was detected. Full data set is reported in supplementary table S1.

Quantitative RT-PCR analyses were performed on both CFX96 Touch (Biorad) and 7900HT (Applied Biosystems) detection systems (both already recommended by CDC guidelines), using the CDC 2019-nCoV rRT-PCR Diagnostic Panel. 
The two systems were analytically validated using the 2019-CoV plasmid control (Integrated DNA Technologies, Inc.), containing the complete nucleocapsid gene (data not reported).

In order to evaluate the RNA extraction efficiency, the internal amplification control (IC) gene ( $\beta$-Actin) was used, comparing data obtained with the adapted-RNeasy method and the validated Roche-Magnapure extraction method on the clinical samples set.

All analyzed samples resulted positive for the internal amplification control (table S1) and the Ct showed a good correlation between the two methods (Fig.1).

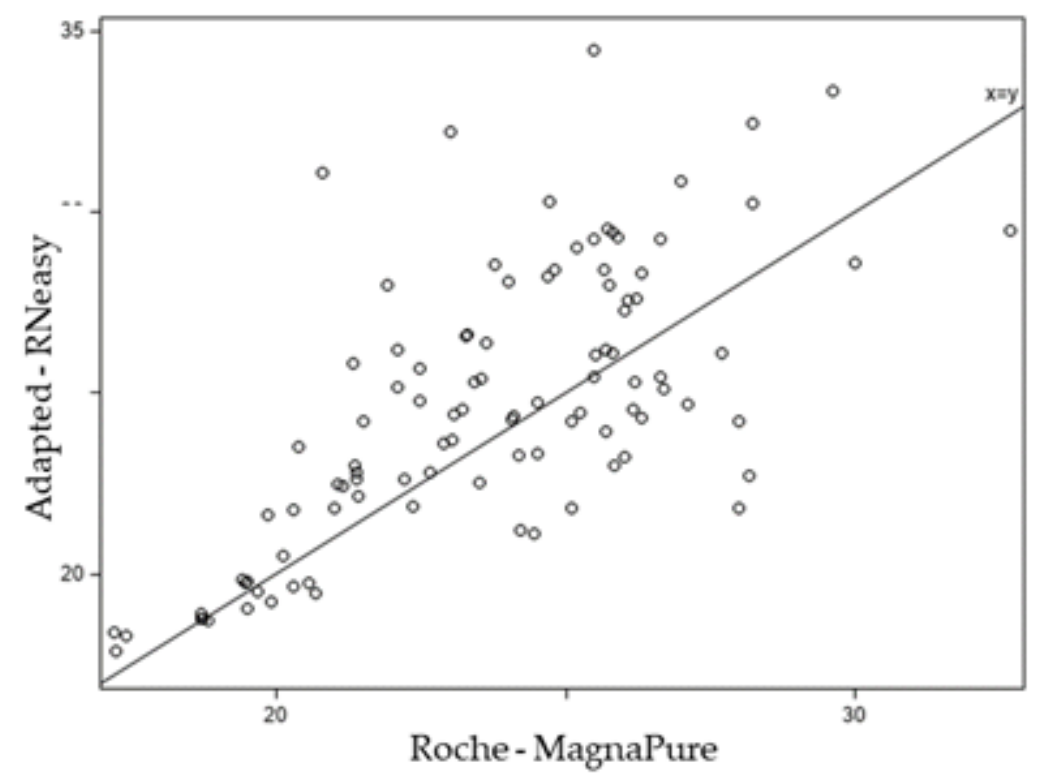

Figure 1. Correlation plot between the raw data signal $(\mathrm{Ct})$ of the $\beta$-Actin control from the two different methods.

The comparison of the mean difference in $\beta$-Actin control was performed to assess how and if the Ct distribution of the samples influences this parameter. As shown in Figure 2 we observed lower variation in the raw data at the lowest $\mathrm{Ct}$ value (left) and higher at highest Ct value (right). Overall, we observed about $1 \mathrm{Ct}$ of difference between samples from adapted-RNeasy method and the validated Roche-Magnapure.

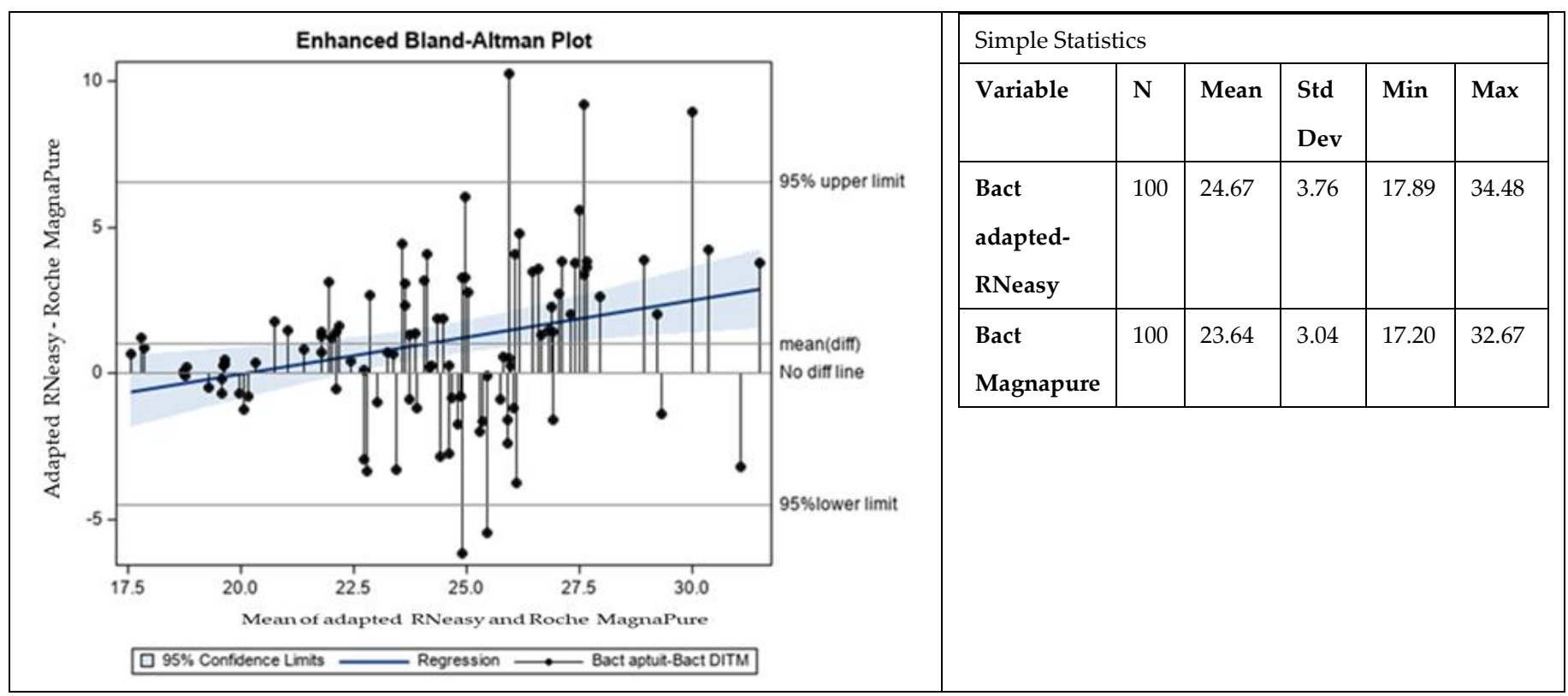


Figure 2. Enhanced Bland-Altman Plot shows the mean differences of $\beta$-Actin between the two methods in the overall raw data $(\mathrm{Ct})$ distribution. The table shows simple statistics of the raw data analyzed.

Data obtained from the difference between the two methods were also plotted on a bar graph that showed the normal distribution of the $\beta$-Actin (Kolmogorov-Smirnov $\mathrm{p}>0.150$, Figure 3).

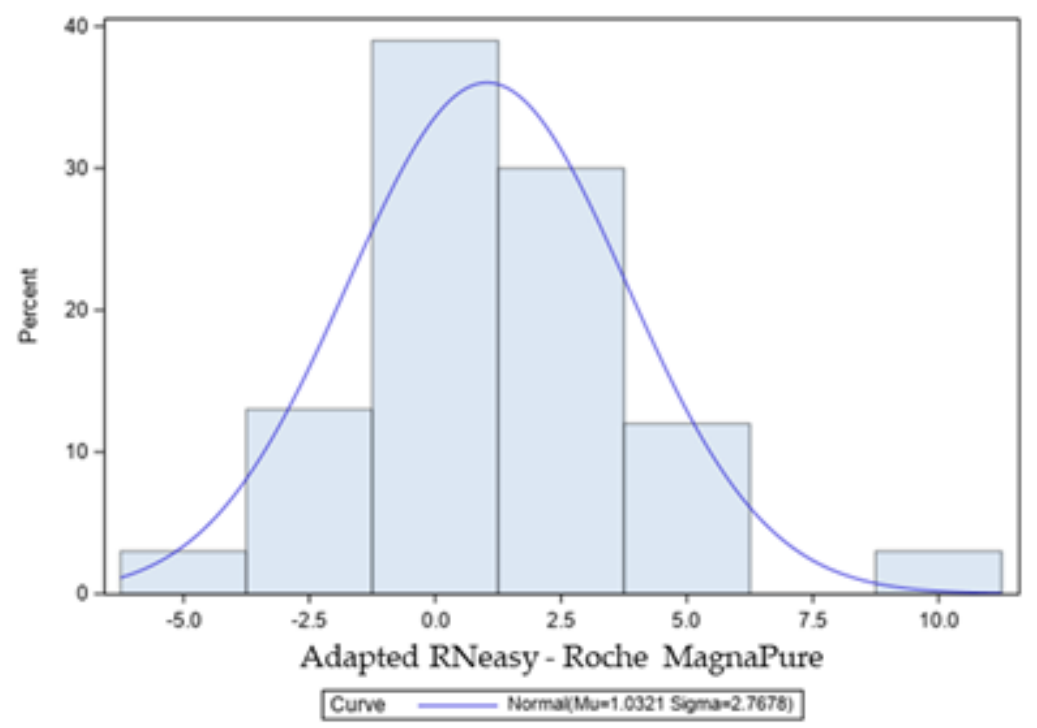

Figure 3. Bar graph showing the distribution of the difference in means of $\beta$-Actin control of the two different methods: as shown here the distribution resembles the Gaussian curve.

The evaluation of the N1 target gene, reported in table 2, showed that the RNeasy-adapted method was able to detect 3 additional positive samples (with high Ct, indicating a low viral load, confirmed by subsequent repeted analysis). Refer to table S1 for the full data set. The mean Ct observed between the two methods was very similar (Delta Ct<1). When evaluating the N2 target gene, the RNeasy-adapted method was able to detect 3 additional positive samples with respect to the magnapure method (with high $\mathrm{Ct}$, indicating a low viral load confirmed by subsequent repeted analysis). Refer to table S1 for the full data set. For N2 target the difference was about $3 \mathrm{Ct}$, indicating an even higher sensitivity for the RNeasy compared to the Magnapure method.

\begin{tabular}{|c|c|c|c|c|c|c|c|c|c|c|c|c|c|}
\hline \multicolumn{7}{|c|}{ Simple Statistics } & \multicolumn{7}{|c|}{ Simple Statistics } \\
\hline Variable & $\mathbf{N}$ & Mean & $\begin{array}{l}\text { Std } \\
\text { Dev }\end{array}$ & Sum & Min & Max & Variable & $\mathbf{N}$ & Mean & $\begin{array}{l}\text { Std } \\
\text { Dev }\end{array}$ & Sum & Min & Max \\
\hline $\begin{array}{l}\mathrm{N} 1- \\
\text { RNeasy- } \\
\text { adapted }\end{array}$ & 19 & 30.94 & 5.67 & 587.81 & 19.43 & 37.09 & $\begin{array}{l}\mathrm{N} 2 \\
\text { RNeasy- } \\
\text { adapted }\end{array}$ & 17 & 30.09 & 6.13 & 511.53 & 17.94 & 36.57 \\
\hline $\begin{array}{l}\text { N1_ } \\
\text { Magnapure }\end{array}$ & 17 & 31.54 & 6.04 & 536.23 & 17.16 & 38.47 & $\begin{array}{l}\mathrm{N} 2 \\
\text { Magnapure }\end{array}$ & 14 & 33.86 & 6.70 & 473.97 & 18.46 & 40.16 \\
\hline
\end{tabular}

Table 1. Summary table with simple statistics of N1 and N2 raw data from positive samples obtained with both methods. 
Finally, the analysis performed using the Cohen's kappa coefficient to assess the agreement in the ratings using positive samples for N1 or N2 genes shows accuracy with an estimate value of 0.90 .

\section{Conclusions}

Despite the differences in the technology on which the two RNA extraction methods are based (magnetic beads vs columns) and in the processing (automated vs manual), the agreement we obtained for the IC and target genes on the analyzed set of clinical samples demonstrated that the two methods can be considered equivalent and that the RNeasyadapted method can be applied for the SARS-CoV-2 diagnostics. The addition of this new extraction method resulted in a throughput increase for SARS-CoV-2 molecular test of about 2000 samples/month in our laboratory during the initial months of the pandemic emergency in which the lack of reagents for the extraction led to an insufficient and slow throughput of the analysis of the swabs. The addition of this extraction method can help in support diagnostic labs, even in reagent scarcity condition and low automation settings.

\section{Materials and Methods}

\section{Setting and participants}

A total of 100 nasopharyngeal swabs samples were used for the new method validation. Samples were collected at the Department of Infectious, Tropical Diseases and Microbiology (DITM) of the IRCCS Sacro Cuore Don Calabria Hospital from subjects referring to the Hospital Emergency Room for COVID-19 suspect. Nasopharyngeal swabs (e-swab, Copan) were stored at $4^{\circ} \mathrm{C}$ till analysis. Each sample was processed in parallel for both the routinely used diagnostic protocol and the new alternative protocol. For each sample, full informed consent was collected from patients/subjects for the use of human biological materials and for data treatment for research purpose and the study was approved by the competent Ethics Committee for Clinical Research of Verona and Rovigo Provinces (Prot n 46404/2020).

\section{RNA extraction and rRT-PCR}

DITM was qualified for SARS-CoV-2 molecular diagnosis by the Regional Reference laboratory (Department of Microbiology, University Hospital of Padua). The routinely used diagnostic protocol, performed on nasopharyngeal swabs, was based on Roche-Magnapure kit (an EUA recommended test) and standardized following the WHO guidelines [12]. Briefly, RNA extraction was performed by an automated method (Magnapure LC RNA isolation kitHigh performance, Roche) according to the manufacturer's instructions. RNA was eluted in elution buffer and rRTPCR test was executed using the CDC 2019-nCoV rRT-PCR Diagnostic Panel assay (Integrated DNA Technologies, Inc., USA) and protocol (https://www.fda.gov/media/134922/download) with a LOD of 10 copies/well, using a CFX96 Touch Real-Time PCR Detection System (Bio-Rad). As internal control the human $\beta$-Actin target was used [13]. As positive control, the 2019-CoV plasmid control (Integrated DNA Technologies, Inc.), containing the complete nucleocapsid gene, was used. A non-template control (NTC) was used as negative control for the reaction. Thermal cycling conditions were $5 \mathrm{~min}$ at $50^{\circ} \mathrm{C}$ for reverse transcription, $20 \mathrm{sec}$ at $95^{\circ} \mathrm{C}$ for RT inactivation/initial denaturation/Taq activation, and 45 cycles of $3 \mathrm{sec}$ at $95^{\circ} \mathrm{C}$ and $30 \mathrm{sec}$ at $58^{\circ} \mathrm{C}$ for the amplification phase. The threshold for the cycle threshold (Ct) analysis was arbitrarily set at 200 (in the exponential amplification phase) for all the runs. A positive result was defined as a curve that crossed the threshold within 45 cycles ( $\mathrm{Ct} \leq 45)$.

For the adapted-RNeasy method, the same samples extracted with the Magnapure method were distributed in deepweel plates and RLT buffer was added. Then the inactivation was completed at $56^{\circ} \mathrm{C}$ for 15 minutes [14]. Plates were maintained at room temperature for the following steps. Briefly, $250 \mu \mathrm{L}$ of $96 \%$ ethanol was added to each sample in the working plate and carefully mixed by pipetting. Samples were successively loaded onto the RNeasy 96 plate and centrifuged at $5600 \mathrm{xg}$ for 4 minutes at room temperature. $350 \mu \mathrm{L}$ of buffer RW1 was added and samples were centrifuged again at $5600 \mathrm{xg}$ for 4 minutes at room temperature. $80 \mu \mathrm{L}$ of DNAse I mix (prepared following manufacturer's instructions) was added to each sample and then the plate was incubated for 15 minutes at room temperature. After DNAse I step the regular procedure for extraction was followed, and RNA was finally eluted using 
$80 \mu \mathrm{L}$ of nuclease free water after 1 minute of incubation. The amplification was performed in 96-well plates on an Applied Biosystems 7900HT PCR Instrument (Thermo Fisher Scientific), replicating the routinely used protocol for the CDC 2019-nCoV rRT-PCR Diagnostic Panel assay and the human $\beta$-Actin target as internal control [13]. The threshold for the 7900HT PCR Instrument was set at 0.05 in the exponential amplification phase in Log view. A positive result was defined as a curve that crossed the threshold within 45 cycles (Ct $\leq 45)$. Analytical sensitivity of up to a LOD of 10 copies/well was verified using the 2019-CoV plasmid control (Integrated DNA Technologies, Inc.).

Table 2 compares volumes used for the two extraction methods applied to nasopharyngeal swabs.

\begin{tabular}{|l|l|l|}
\hline & Adapted-RNeasy (Qiagen) & Magnapure (Roche) \\
\hline Input (Nasopharyngeal Swab) & $100 \mu \mathrm{L}$ & $200 \mu \mathrm{L}$ \\
\hline Dilution Reagent & $350 \mu \mathrm{L}$ RLT + 1\% DTT & $+1 \% \mathrm{DTT}$ \\
\hline Final Elution Volume & $80 \mu \mathrm{L}$ & $100 \mu \mathrm{L}$ \\
\hline Input For The rRT-PCR Analysis & $\begin{array}{l}10 \mu \mathrm{L} / 80 \mu \mathrm{L} \text { (or } 5 \mu \mathrm{L} \text { for the IC } \\
\text { analysis) }\end{array}$ & $\begin{array}{l}10 \mu \mathrm{L} / 100 \mu \mathrm{L} \text { (or } 5 \mu \mathrm{L} \text { for the IC } \\
\text { analysis) }\end{array}$ \\
\hline Heat Inactivation & $56^{\circ} \mathrm{C}$ for 15 minutes & None \\
\hline
\end{tabular}

Table 2. Summary table of samples input, dilution and elution volumes of both extraction methods.

\section{Statistical analysis}

We compared laboratory methods using an enhanced version of Bland-Altman plots to analyze means differences. The association between results was also visually inspected using Scatterplots by evaluating their distance from the identity line. We also computed simple statistics and Cohen's kappa coefficient to analyze the distribution of the data and the agreement in the ratings, respectively. The data analysis was performed using SAS software (SAS Institute Inc 2013. SAS/STATA® 9.4).

Supplementary Materials: The following are available online at www.mdpi.com/xxx/s1, Table S1: Data set.

Author Contributions: EB, FR, CP: conceptualization, method execution, data analysis and discussion and paper writing. MP, CC, MP, SS, ML: method execution. CG, RR, FF, FP: results discussion and paper revision. RS: statistical analysis. All authors have read and agreed to the published version of the manuscript." Please turn to the CRediT taxonomy for the term explanation. Authorship must be limited to those who have contributed substantially to the work reported.

Acknowledgment: the authors would like to thank Federico Faggioni for the laboratory support.

Funding: This research was funded by Italian Ministry of Health “Fondi Ricerca Corrente, Project L1P6" for IRCCS Sacro Cuore Don Calabria Hospital.

Institutional Review Board Statement: The study was conducted according to the guidelines of the Declaration of Helsinki, and approved by the Ethics Committee for Clinical Research of Verona and Rovigo Provinces (Prot n 46404/2020)

Informed Consent Statement: Informed consent was obtained from all subjects involved in the study.

Data Availability Statement: all the data are available as supplementary material (Table S1)

Conflicts of Interest: The authors declare no conflict of interest.

\section{References}

1. WHO. Laboratory testing for 2019 novel coronavirus in suspected human cases. Technical guidance. . In https://www.who.int/emergencies/diseases/novel-coronavirus-2019/technicalguidance. , 2020.

2. Prevention, C.f.D.C.a. Clinical specimens: novel coronavirus (2019-nCoV). . In https://www.cdc.gov/coronavirus/2019ncov/lab/guidelines-clinical-specimens.html, 2020. 
3. Lubke, N.; Senff, T.; Scherger, S.; Hauka, S.; Andree, M.; Adams, O.; Timm, J.; Walker, A. Extraction-free SARS-CoV-2 detection by rapid RT-qPCR universal for all primary respiratory materials. Journal of clinical virology : the official publication of the Pan American Society for Clinical Virology 2020, 130, 104579, doi:10.1016/j.jcv.2020.104579.

4. Bruce, E.A.; Huang, M.L.; Perchetti, G.A.; Tighe, S.; Laaguiby, P.; Hoffman, J.J.; Gerrard, D.L.; Nalla, A.K.; Wei, Y.; Greninger, A.L., et al. Direct RT-qPCR detection of SARS-CoV-2 RNA from patient nasopharyngeal swabs without an RNA extraction step. PLoS biology 2020, 18, e3000896, doi:10.1371/journal.pbio.3000896.

5. Alcoba-Florez, J.; Gonzalez-Montelongo, R.; Inigo-Campos, A.; de Artola, D.G.; Gil-Campesino, H.; The Microbiology Technical Support, T.; Ciuffreda, L.; Valenzuela-Fernandez, A.; Flores, C. Fast SARS-CoV-2 detection by RT-qPCR in preheated nasopharyngeal swab samples. International journal of infectious diseases : IJID : official publication of the International Society for Infectious Diseases 2020, 97, 66-68, doi:10.1016/j.ijid.2020.05.099.

6. Merindol, N.; Pepin, G.; Marchand, C.; Rheault, M.; Peterson, C.; Poirier, A.; Houle, C.; Germain, H.; Danylo, A. SARS-CoV-2 detection by direct rRT-PCR without RNA extraction. Journal of clinical virology : the official publication of the Pan American Society for Clinical Virology 2020, 128, 104423, doi:10.1016/j.jcv.2020.104423.

7. Guan, B.; Frank, K.M.; Maldonado, J.O.; Beach, M.; Pelayo, E.; Warner, B.M.; Hufnagel, R.B. Sensitive extraction-free SARSCoV-2 RNA virus detection using a novel RNA preparation method. medRxiv : the preprint server for health sciences 2021, 10.1101/2021.01.29.21250790, doi:10.1101/2021.01.29.21250790.

8. Waggoner, J.J.; Stittleburg, V.; Pond, R.; Saklawi, Y.; Sahoo, M.K.; Babiker, A.; Hussaini, L.; Kraft, C.S.; Pinsky, B.A.; Anderson, E.J., et al. Triplex Real-Time RT-PCR for Severe Acute Respiratory Syndrome Coronavirus 2. Emerging infectious diseases 2020, 26, 1633-1635, doi:10.3201/eid2607.201285.

9. Nalla, A.K.; Casto, A.M.; Huang, M.W.; Perchetti, G.A.; Sampoleo, R.; Shrestha, L.; Wei, Y.; Zhu, H.; Jerome, K.R.; Greninger, A.L. Comparative Performance of SARS-CoV-2 Detection Assays Using Seven Different Primer-Probe Sets and One Assay Kit. Journal of clinical microbiology 2020, 58, doi:10.1128/JCM.00557-20.

10. Perchetti, G.A.; Nalla, A.K.; Huang, M.L.; Zhu, H.; Wei, Y.; Stensland, L.; Loprieno, M.A.; Jerome, K.R.; Greninger, A.L. Validation of SARS-CoV-2 detection across multiple specimen types. Journal of clinical virology : the official publication of the Pan American Society for Clinical Virology 2020, 128, 104438, doi:10.1016/j.jcv.2020.104438.

11. Dong, E.; Du, H.; Gardner, L. An interactive web-based dashboard to track COVID-19 in real time. The Lancet. Infectious diseases 2020, 20, 533-534, doi:10.1016/S1473-3099(20)30120-1.

12. WHO. Laboratory testing for coronavirus disease (COVID-19) in suspected human cases: interim guidance, 19 March 2020. https://apps.who.int/iris/handle/10665/331501, 2020.

13. Imwong, M.; Hanchana, S.; Malleret, B.; Renia, L.; Day, N.P.; Dondorp, A.; Nosten, F.; Snounou, G.; White, N.J. Highthroughput ultrasensitive molecular techniques for quantifying low-density malaria parasitemias. Journal of clinical microbiology 2014, 52, 3303-3309, doi:10.1128/JCM.01057-14.

14. Darnell, M.E.; Subbarao, K.; Feinstone, S.M.; Taylor, D.R. Inactivation of the coronavirus that induces severe acute respiratory syndrome, SARS-CoV. Journal of virological methods 2004, 121, 85-91, doi:10.1016/j.jviromet.2004.06.006. 